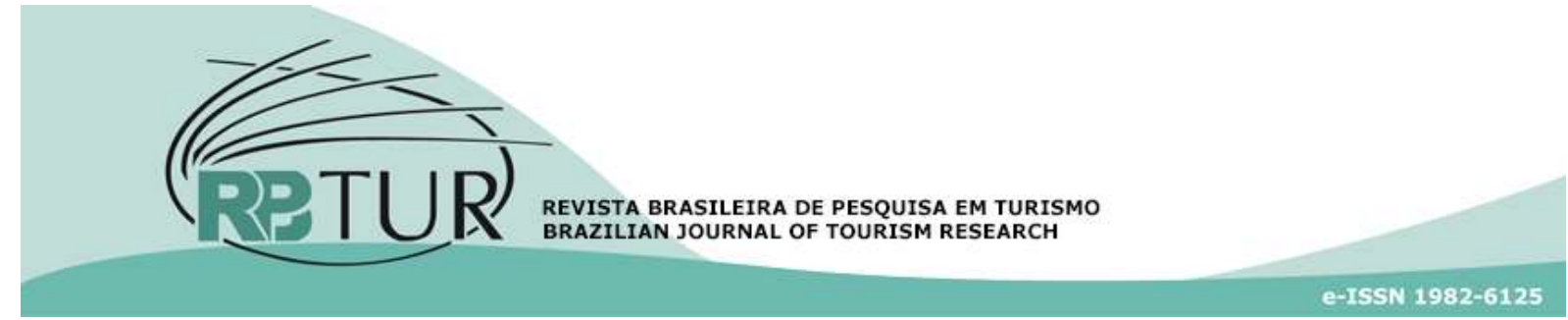

Artigo

DOI: http://dx.doi.org/10.7784/rbtur.v11i1.1201

\title{
Os elementos que caracterizam o Cluster Turístico em Balneário Camboriú, Santa Catarina, Brasil
}

\author{
The elements that characterized the Tourism Cluster in Balneario \\ Camboriu, Santa Catarina, Brazil
}

\section{Los elementos que caracterizan el Cluster de Turismo en Balneário Camboriú, Santa Catarina, Brasil}

\author{
Ana Paula Sohn ${ }^{1}$ \\ Carolina Silvestrini ${ }^{2}$ \\ Thamires Foletto Fiuza ${ }^{3}$ \\ Pablo Flôres Limberger ${ }^{4}$
}

Resumo: O turismo promove o desenvolvimento regional que pode ser impulsionado por meio da formação de redes interorganizacionais como os clusters. Os clusters turísticos são compostos por instituições e organizações numa mesma região geográfica que almejam melhorar a sua competitividade. A perspectiva analítica dos clusters turísticos ganha cada vez mais importância no debate sobre desenvolvimento regional. Diante deste contexto a pesquisa tem como objetivo central descrever e analisar os elementos que caracterizam a constituição de um cluster turístico em Balneário Camboriú, Santa Catarina, Brasil. Para a identificação dos elementos constituintes do cluster realizou-se uma pesquisa exploratória, bibliográfica e documental. O modelo teórico de análise da maturidade adotado foi o proposto por Cunha (2003), este modelo foi escolhido porque incorpora critérios utilizados pela EURADA em suas pesquisas para classificação e avaliação do desempenho de aglomerados produtivos na União Européia. Por meio da metodologia aplicada, Balneário Camboriú (SC) é considerado um

\footnotetext{
1 Universidade do Vale do Itajaí (UNIVALI), Balneário Camboriú, SC, Brasil. Concepção e desenho do trabalho científico; interpretação dos dados; redação e revisão crítica do trabalho.

${ }^{2}$ Universidade do Vale do Itajaí (UNIVALI), Balneário Camboriú, SC, Brasil. Pesquisa em base de dados, redação do corpo teórico.

${ }^{3}$ Universidade do Vale do Itajaí (UNIVALI), Balneário Camboriú, SC, Brasil. Pesquisa em base de dados, redação do corpo teórico, aquisição dos dados.

${ }^{4}$ Universidade do Vale do Itajaí (UNIVALI), Balneário Camboriú, SC, Brasil. Revisão crítica do trabalho.
}

Artigo recebido em: 16/08/2016 Artigo Aprovado: 16/11/2016 
cluster turístico que promove por meio da cooperação entre os atores ganhos coletivos e o desenvolvimento do setor na região.

Palavras-chave: Cluster Turístico. Turismo. Desenvolvimento Regional. Balneário Camboriú.

Abstract: Tourism for its growth potential and for being a product that can only be consumed on-site promotes regional development which can be boosted by the formation of clusters. The tourism clusters are composed of institutions and organizations in the same geographic region that aim to improve their competitiveness. The analytical perspective of tourism clusters is gaining increasing importance in the debate on regional development. The city of Balneário Camboriú is recognized as the Santa Catarina Capital of Tourism due to the importance achieved by the sector. Given this context, the research aims to describe and analyze the elements that characterize the establishment of a tourist cluster in Balneário Camboriú, Santa Catarina, Brazil. For the identification of the cluster elements was carried out an exploratory research, bibliographic and documental. The theoretical model of maturity analysis adopted was proposed by Cunha (2003), this model was chosen because it incorporates criteria used by EURADA in their research for classification and evaluation of the performance of productive clusters in the European Union. Through the methodology applied, Balneário Camboriú (SC) is considered a tourist cluster that promotes through cooperation between actors collective gains and the development of the sector in the region.

Keywords: Tourist Cluster. Tourism. Regional Development. Balneário Camboriú.

Resumen: El turismo promueve el desarrollo regional que puede ser accionado a través de la formación de redes entre organizaciones como clusters. Los grupos turísticos se componen de instituciones y organizaciones en la misma región geográfica que tiene como objetivo mejorar su competitividad. La perspectiva analítica de los Clusters de Turismo gana cada vez más importancia en los debates acerca del desarrollo regional.Teniendo en cuenta este contexto, la investigación tiene como objetivo describir y analizar las características de la creación de un cluster de turismo en Balneário Camboriú, Santa Catarina, Brasil. Para la identificación de los constituyentes de cluster, fue celebrada una investigación exploratoria, en la literatura y documentos. El modelo teórico de análisis de madurez adoptado, fue el propuesto por Cunha (2003), este modelo se ha elegido porque incorpora criterios utilizados por EURADA en sus investigaciones para la clasificación y evaluación del desempeño de los aglomerados de producción en la Unión Europea. A través de la metodología aplicada, Balneário Camboriú (SC) es considerado un cluster turístico que promueve a través de la cooperación de los actores, ganancias colectivas y el desarrollo del sector en la región.

Palabras clave: Cluster de Turismo. Turismo. Desarrollo Regional. Balneário Camboriú.

\section{INTRODUÇÃO}

O turismo é considerado um dos mais importantes setores da economia global e estratégico para o desenvolvimento regional (Jackson \& Murphy, 2006; Mabrouk, McDonald, Mocan, \& Summa, 2008; Ferreira \& Estevao, 2009; Aleksandrova, 2016). É multifacetado e engloba diversos atores e que têm no território importante fonte de vantagem competitiva. Sendo um setor que contribui diretamente para o desenvolvimento regional por meio da formação de redes interorganizacionais como os clusters (Kunz, Schommer, Schneider, \& Mecca, 2012; Silva, 2015).

Devido as vantagens alcançadas pela criação de clusters turísticos, tem sido dada uma crescente atenção para políticas de criação e competitividade de clusters por parte das entidades públicas, de organizações como a Organização Mundial do Turismo (OMT) e por parte da academia. Pode-se destacar que as pesquisas sobre esta 
temática voltam-se para o entendimento das oportunidades e vantagens da formação de redes interorganizacionais regionais como os clusters turísticos (Novelli, Schmitz, \& Spencer, 2006; Jackson \& Murphy, 2006; Ferreira \& Estevao, 2009; lordache, Ciochinã, \& Asandei, 2012; Kunz et al., 2012; Borkowska-Niszczota, 2015; Aleksandrova, 2016). Para Fundeanu (2015), a clusterização permite a junção de esforços entre as empresas do setor turístico, e estimula uma maior articulação com outras instituições. $A$ articulação com estas categorias de instituições, que se torna mais estreita nos clusters, estimula os processos de inovação (Sohn, Vieira, Casarotto Filho, Cunha, \& Zarelli, 2016), considerados um dos fatores fundamentais para competitividade no turismo (Novelli et al., 2006)

Para a Organização Mundial do Turismo [OMT] (n.d.) a constituição de clusters turísticos é uma tendência em curso em diversos países com o objetivo de promover o desenvolvimento regional e de estimular a inovação, a competitividade e o desenvolvimento econômico e social. Assim destaca-se que o debate acerca dos clusters turísticos é estimulado por programas de desenvolvimento local como estratégia adequada para combater as disparidades regionais e desigualdades sociais (Borkowska-Niszczota, 2015; Yıldız \& Aykanat, 2015; Martins, Fiates, \& Pinto, 2016; Polukhina, 2016).

Diante deste contexto a pesquisa tem como objetivo central descrever e analisar os

\footnotetext{
${ }^{5}$ O período considerado alta temporada em Balneário Camboriú ocorre entre novembro e janeiro, sendo caracterizado pelo forte calor provocado pelo
}

elementos que caracterizam a constituição de um cluster turístico em Balneário Camboriú, Santa Catarina, Brasil.

A cidade de Balneário Camboriú está localizada no litoral de Santa Catarina, Brasil, apresentando a partir da segunda metade do século XX uma vocação para indústria do turismo. O município que possui cerca de 128.155 mil habitantes (Instituto Brasileiro de Geografia e Estatística [IBGE], 2015), e recebeu 600 mil visitantes por mês durante o período de alta temporada ${ }^{5}$ no ano de 2015 (Prefeitura Municipal de Balneário Camboriú, 2016).

Para atender ao objetivo proposto o artigo está organizado em cinco seções. $\mathrm{Na}$ introdução tem-se a apresentação do contexto, objetivo e importância da pesquisa, em seguida a base teórica, a metodologia, os resultados e as considerações finais.

\section{CLUSTER: FUNDAMENTOS CONCEITUAIS}

Clusters são um conjunto de organizações que atuam em um mesmo setor, localizadas em uma mesma região geográfica e interligados por meio de relações 'comprador e fornecedor' e 'fornecedor e comprador', ou por tecnologia de propriedade comum, compradores comuns ou o mesmo canal de distribuição ou concentração de trabalhadores (Cunha, 2007). Considera-se que os clusters são concentrações geográficas de empresas e instituições interconectadas em um campo ou setor particular que englobam uma

verão no município, aliado ao período de férias escolares. 
coleção de indústrias e entidades vitais para a competição (Porter, 2008; Sölvell, 2008).

As definições apresentadas de cluster pressupõe a localização dentro de um mesmo território geográfico de diferentes agentes que trabalham em prol de um determinado setor econômico (figura 1). Dentre estes agentes destaca-se a presença de associações de suporte privadas ou ligadas ao governo (Casarotto \& Pires 2001).

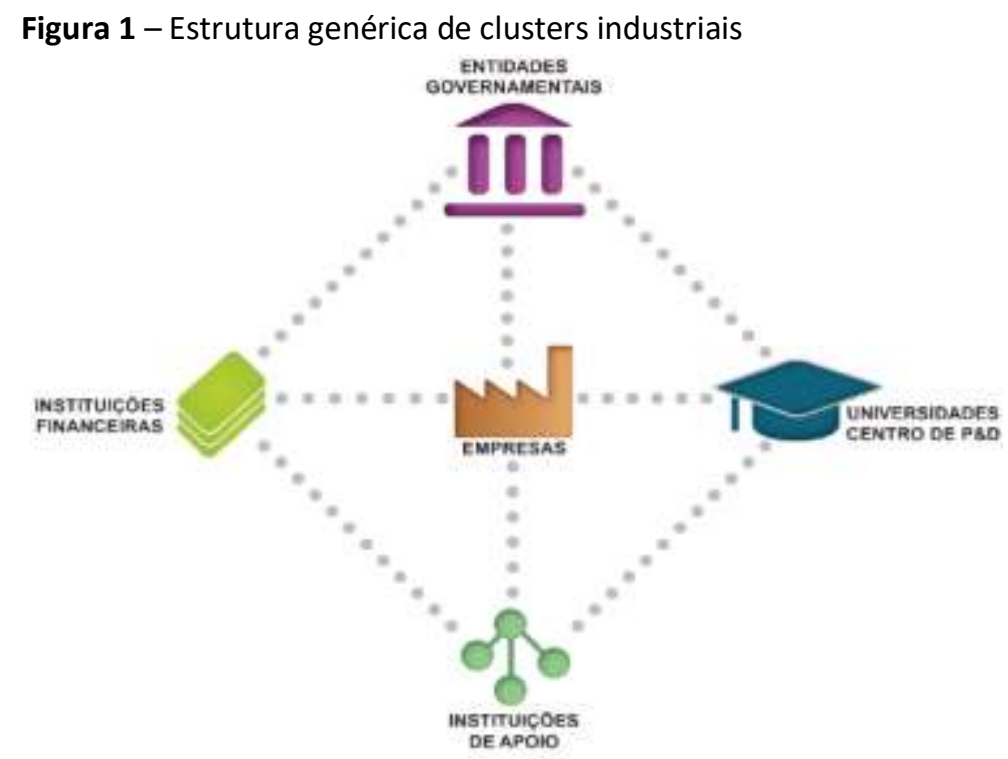

Fonte: Adaptado de Sölvell (2008)

Autores como Casarotto e Pires (Yildiz \& Aykanat, 2015). Um cluster (2001), Belso-Martínez (2006), Cunha (2007), compreende-se através de uma entidade Jenkis e Tallman (2010), Lundberg (2010), socioeconômica caracterizada pela Porter e Kramer (2011), Saublens (2011), comunidade social de agentes que trabalham Wragg (2012), Li et al. (2012) e Yildiz e juntos em atividades econômicas Aykanat (2015), destacam a importância dos interligadas, fazendo-se uso do clusters para a promoção da competitividade conhecimento tecnológico e organizacional a e do desenvolvimento regional. Nesta fim de gerar produtos e serviços mais perspectiva, salienta-se que a formação de competitivos (Morosini, 2004; Porter, 2008). clusters é importante para toda economia regional que prospera atinge relevantes taxas de crescimento.

A existência de clusters promove a geração de externalidades (Waxell \& Malmberg, 2007; Nie \& Sun, 2015) por meio do desenvolvimento de parcerias interorganizacionais a fim de atingir um elevado nível de inovação e competitividade Praticamente todas as correntes de pensamento sobre as aglomerações produtivas valorizam a importância da cooperação (Martins et al., 2016) e que ocorre em diferentes dimensões e pode gerar ganhos, entre os quais: aumento da produtividade, aumento do ritmo de inovação e estímulo na criação de novos negócios (Lai, Hsu, Lin, Chen, \& Lin, 2014; Yil- 
diz \& Aykanat, 2015).

A participação de empresas em clusters contribui na adaptação a mercados, em mudança rápida e aquisição de novas tecnologias, pois, empresas localizadas em clusters podem trabalhar de forma colaborativa para co-evoluirem com o objetivo de aumentar a competitividade e adaptação à mudança (Yildiz \& Aykanat, 2015; Lai et al., 2014; Niu, 2010).

As interações sociais e os vínculos produzidos no interior dos clusters se convertem em determinantes-chave para o intercâmbio de conhecimento e para a geração de externalidades tecnológicas relacionadas a inovação (Hortelano, Pérez \& Villaverde, 2015; Sohn et al., 2016).

Todavia, ao evidenciarem a importância das competências locais para a competitividade dos clusters Porter e Kramer (2011), alertam para o fato de que deficiências nas condições estruturais oneram as empresas. Desta forma, problemas relacionados à discriminação racial, pobreza, degradação ambiental e trabalhadores sem saúde podem deixar a empresa cada vez menos conectada à comunidade, diminuindo sua influência na resolução destes e de outros problemas. Apontam ainda que, a manutenção da competitividade no âmbito dos clusters está relacionada ao investimento no desenvolvimento de tecnologias de produtos e processos.

Observa-se a presença de um fluxo de informações, know how, e conhecimentos no cluster entre as organizações que fazem parte de clusters. Este fluxo se dá por meio de canais de transmissão (Sohn et al., 2016) que promovem o Local Knowledge Spillovers (LKS). Os LKS são os ganhos em conhecimento por meio da troca de informações sem compensação à fonte de conhecimento, também podendo ser induzidos por meio de interações intencionais (Kesidou \& Romijn, 2008).

A partir de estudos sobre a capacidade de absorção de um cluster de vinho no Chile a autora Elisa Giuliani (2011) diz que a localização de atividades especializadas produzem economias externas, geradas pela presença de três fatores: disponibilidade de inputs locais, mão de obra qualificada, e spillovers de conhecimento ${ }^{6}$. Nesse contexto, Giuliani (2011) apresenta o conceito de Innovative milieu, uma rede de relações sociais informais em uma área geográfica limitada que realça a capacidade inovativa por meio de processos de aprendizagem coletiva. O aprendizado coletivo é um processo social no qual os mecanismos da transferência de conhecimento são sociais porque novos conhecimentos são transferidos para outros agentes, graças a rotina tecnológica, organizacional, e institucional e comportamentos que facilitam o compartilhamento de know-how e informações.

Partindo desse princípio de difusão de conhecimento, é possível identificar e analisar a "capacidade de absorção" do cluster, que, para Giuliani (2011) pode ser

\footnotetext{
${ }^{6}$ Spillovers do conhecimento envolve o derramamento do conhecimento sobre as pessoas, sobre o tecido empresarial e organizacional e sobre o espaço local.
} 
considerado a capacidade interna da empresa de reconhecer os novos conhecimentos que provém do ambiente externo, e aplicálos para fins comerciais, ou seja, uma capacitação em absorver, difundir e explorar criativamente todo o conhecimento adquirido por fontes externas ao cluster. As empresas, instituições, centros de pesquisa, universidades e outras organizações que fazem parte de um cluster e estão expostos a essas fontes externas de conhecimento são chamados gatekeepers de conhecimento. Os gatekeepers de conhecimento são organizações e/ou indivíduos que se diferenciam das demais ao terem contato com fontes de informações técnicas mais avançadas desenvolvidas em outras localidades, ou seja, filtram informações e conhecimento externos ao cluster. Dependendo do contexto, podem ser entendidos também como firmas que transferem conhecimentos extra-cluster para o sistema intra-cluster. São organizações capazes de traduzir o conhecimento adquirido em know-how que pode ser aproveitado por outras organizações localizadas no cluster.

A formação de ligações com fontes externas, e as características estruturais internas do cluster, são outros dois fatores que estão inter-relacionados que compõem a capacidade de absorção, caracterizando-a como a capacidade de estabelecer as ligações intra e extra cluster. Os quais são importantes para o crescimento da produtividade e estão presentes junto dos compradores, fornecedores, e outras instituições, contribuindo para o desenvolvimento (Giuliani, 2011; Graf, 2011).

Nos estudos sobre clusters, existem dois pontos na análise do ciclo de vida que merecem atenção: um deles é o do grau de desenvolvimento alcançado; o outro, é o tempo para o seu amadurecimento, desde os estágios que predominam a informalidade, até o nível máximo de eficiência em que imperam os sistemas inovativos (Cunha, 2003).

Para Casarotto e Pires (2001), o cluster desenvolve-se sobre a vocação regional e pode verticalizar-se a jusante (serviços) ou a montante (fornecedores), passando por diferentes fases ao longo do seu ciclo de vida conforme apresentado no quadro 1.

Quadro 1 - Ciclo de vida de um cluster

\begin{tabular}{|l|l|}
\hline Estágio de desenvolvimento & Características \\
\hline Pré-cluster & Poucas empresas isoladas voltadas a um mesmo produto/serviço \\
\hline Nascimento do cluster & Maior concentração de empresas e fortes relações comerciais \\
\hline Desenvolvimento do cluster & $\begin{array}{l}\text { Aumento da concentração com verticalização e início da formação de } \\
\text { consórcios }\end{array}$ \\
\hline Cluster estruturado & Sistema local estruturado, forte parceria público-privada \\
\hline
\end{tabular}

Fonte: adaptado de Casarotto e Pires (2001); Menzel e Fornahl (2009)

Sob a perspectiva da criação de sistemas inovativos e com base em indicadores propostos pela EURADA, Cunha
(2003) apresenta um recorte analítico para análise do ciclo de vida de clusters que foi utilizado como metodologia de análise para 
esta pesquisa. Segundo Cunha (2003) há quatro categorias de clusters sintetizados a seguir: a) cluster informal: com predominância de empresas de pequeno porte, com baixa qualificação dos atores, adoção de tecnologias rudimentares, sem representatividade no mercado externo. 0 desempenho econômico é sofrível e inconstante, com empresas que competem marginalmente, e possuem pouca propensão a cooperação, assim não há o entrelaçamento entre firmas o que limita a geração de ganhos resultantes da aproximação espacial e a especialização produtiva das empresas; b) cluster em estágio intermediário: constituído basicamente de empresas de pequeno e médio porte, podendo existir e as tecnologias adotadas são relativamente atualizadas. Há posições variáveis quanto ao desempenho e a propensão à cooperação é baixa, assim como o entrelaçamento entre firmas e a efetiva cooperação; c) cluster em estágio organizado: acolhe uma grande diversidade de estruturas e características empresariais, porém os atores críticos adotam práticas de gestão modernas, com tecnologias atualizadas e, embora haja prevalência de pequenas e médias empresas é freqüente a presença de gatekeepers e de spillovers de conhecimento. Os níveis e potencial de cooperação são médios, com iniciativas de desverticalização, porém ainda insuficientes para gerar ganhos em flexibilidade produtiva e eficiência operacional; d) clusters inovativos: os requisitos para o enquadramento neste estágio são muito rigorosos, pois pressupõem a desverticalização da produção, a abertura de canais de informação, o spillover de conhecimento e um elevado grau de sinergia entre os diferentes atores do aglomerado.

\subsection{Cluster Turístico}

O cluster turístico é composto por um grupo de companhias e instituições relacionadas ao produto de turismo e localizadas em uma mesma região geográfica que buscam por meio da ação conjunta a obtenção de ganhos coletivos (Beni, 2003; Martins et al., 2016). Tem-se nos clusters turísticos a ocorrência de relações do tipo verticais e horizontais entre os envolvidos diretamente e indiretamente com o setor do turismo (Ashton, Valduga, \& Tomazzoni 2015; Tomazzoni, 2015)

Os clusters turísticos impactam positivamente a competitividade das regiões de três formas: 1) aumentando a produtividade das empresas em uma região geográfica, promovendo 0 acesso a provedores e trabalhadores, a informação especializada por meio das relações interorganizacionais; 2) demarcando a direção e ritmo da inovação ao identificar necessidades específicas não atendidas ou pobremente atendidas; 3 ) estimulando novos negócios (conhecimento no tempo real e antecipação das tendência de mercado, conservação entre as várias instituições no cluster e flexibilidade e rápida resposta) (lordache et al., 2010).

Visando entender a relação entre políticas de cluster para o turismo e competitividade regional é proposto por Ferreira e Estevão (2009) um modelo de análise do sistema turístico interativo com 
três componentes principais: 1) produto turístico, 2) a destinação turística e 3) o cluster turístico. $\mathrm{O}$ modelo proposto reconhece o papel dos governantes que definem as políticas que afetam a competitividade do cluster turístico e destaca o papel das universidades como estratégia chave para pesquisas no desenvolvimento da inovação e a diferenciação na oferta de produtos turístico e serviços, assim como, no treinamento e educação nos recursos humanos. É ressaltado pela Polukhina (2016), a necessidade do governo em apoiar as iniciativas nos centros turísticos com as melhores perspectivas. Estes centros tem a capacidade de estimular a economia de um país e desenvolver uma base de suporte para outras iniciativas de clusters.

Fazem parte de clusters turístico: agentes de viagem, guias, fornecedores da indústria de turismo de lazer nacional e local, companhias de transporte, universidades, instituições de treinamento, e outras organizações relevantes trabalhando em conjunto, porém como concorrentes, sendo o objetivo principal dessa cooperação a vantagem competitiva (Cunha \& Cunha, 2005; Martins et al., 2016). O cluster turístico se diferencia das demais modalidades de clusters, pois encontra-se relacionado à oferta de serviços, ou seja, a diferença básica recai no produto final do cluster.

O desenvolvimento do cluster turístico é afetado por vários fatores, como a infraestrutura das atrações turísticas, o tráfego do turismo, e setores de suporte e relacionados (Tomazzoni, 2015; Martins et al., 2016). Entretanto, o fator mais importante é a cooperação de autoridades locais, instituições de apoio, instituições cientificas de pesquisa com empresas locais de pequeno e médio porte (Yildiz \& Aykanat, 2015), sendo que cada membro é responsável por uma determinada tarefa na estrutura do cluster.

As organizações públicas possuem tarefas importantes a desempenhar no estímulo ao desenvolvimento de clusters turísticos, pois é um importante agente em prol da promoção da região turística. Destaca-se neste sentido, a sensibilização e o contato com as empresas visando a criação e o fortalecimento de plataformas para cooperação. Realça-se a importância das organizações públicas no estímulo à ação conjunta para a geração de conhecimento, inovações e introdução de mudanças no sistema educacional para preparar novas formas de colaboração (BorkowskaNiszczota, 2015; Tomazzoni, 2015).

Os impactos positivos que decorrem da existência de clusters na indústria do turismo incluem a especialização das empresas, os efeitos de escala, a melhoria das competências no nível da prestação de serviços e uma articulação mais proveitosa entre a competição e cooperação entre as empresas, a designada coopetição (Santos \& Cerdeira, 2013). Para além destes, salientamse outros aspectos positivos, como: o processo de aprendizagem que resulta da interação mais estreita entre as empresas, a obtenção de spillovers baseados no conhecimento (Cunha \& Cunha, 2005; Souza \& Gil, 2015). Os spillovers de conhecimento ampliam a competência tecnológica da região turística e das organizações ali localizadas (Souza \& Gil, 2015). 


\section{PROCEDIMENTOS METODOLÓGICOS}

Utilizou-se de uma pesquisa exploratória, que segundo Gerhardt e Silveira (2009) busca familiarizar o pesquisador com o tema, buscando torná-lo mais explícito e ainda, possibilitar a construção de hipóteses. Assim, a pesquisa exploratória normalmente é realizada por meio de pesquisas bibliográficas e estudo de caso (Prodanov \& Freitas, 2013).

No que se refere a abordagem a pesquisa utiliza o método qualitativo que "não se preocupa com representatividade numérica, mas, sim, com o aprofundamento da compreensão de um grupo social, de uma organização" (Gerhardt \& Silveira, 2009, p. 31).

Lubeck, Wittmann e Silva (2012) indicam a relevância do uso da metodologia qualitativa em pesquisas sobre identificação e classificação de clusters. Os autores realçam a importância da apresentação informações que demonstrem a existência, a eficiência e eficácia de atividades cooperadas entre empresas.

O presente estudo buscou levantar evidencias de que existe uma concentração de empresas e outras organizações, bem como, uma infraestrutura voltada par o turismo na cidade de Balneário Camboriú. Para alcançar tal objetivo, utilizou-se como base os conceitos de cluster através do enfoque da localização geográfica e da especialização produtiva (Porter, 2008; Sölvell, 2008; Nie \& Sun, 2015) e dos aspectos associados a especialização no setor turístico (Tomazzoni, 2015; Martins et al., 2016; Polukhina, 2016).
Para efetuar descrição dos elementos constitutivos do cluster de Balneário Camboriú, utilizou-se como procedimentos técnicos, uma pesquisa bibliográfica e documental. A pesquisa bibliográfica a fim de buscar referências teóricas sobre o tema, que já foram publicadas, alcançando um maior número de informações e conhecimento prévio sobre o problema que se busca a resposta (Gerhardt \& Silveira, 2009) já, a pesquisa documental foi utilizada visando aprimorar e complementar a pesquisa bibliográfica, através de materiais sem tratamento estatístico (Gerhardt \& Silveira, 2009).

Para o levantamento de dados foram realizados contatos com: 1) a Secretaria Municipal de Turismo e Desenvolvimento Econômico de Balneário Camboriú (SECTUR); 2) o Sindicato de Hotéis, Restaurantes, Bares e Similares de Balneário Camboriú (SINDISOL); e 3) a Universidade do Vale do Itajaí (UNIVALI), entidades ligadas ao setor turístico em Balneário Camboriú. Outras informações foram coletadas por meio de pesquisas em relatórios de organizações nacionais e internacionais como o Programa de Desenvolvimento das Nações Unidas. O roteiro elaborado pelos pesquisadores para a coleta de dados considerava informações sobre: 1) meios de hospedagem, 2) serviços de alimentação e bebidas, 3) agências de viagem; 4) infraestrutura para realização de eventos, 5) presença de centros de formação (universidades, faculdades, centros de treinamento e capacitação e centros de pesquisa) e; 6) número de empregos ligados ao setor de turismo e a remuneração média por pessoa. A coleta e análise destes dados 
ocorreram entre os meses de março e maio de 2016.

Considerando a importância da cooperação e dos ganhos coletivos no interior dos clusters apresentada pela literatura (Giuliani, 2011; Sohn et al., 2016) faz-se a apresentação e análise de dois eventos relacionados ao setor do turismo realizados na cidade. Foram estudados os casos do Balneário Saboroso e do Fórum Científico de Gastronomia, Turismo e Hotelaria (FCGTURH). A seleção destes eventos deu-se devido a importância dos mesmos para o setor e a distinção entre eles. O primeiro é um evento voltado para empresas que atuam na área da gastronomia, sendo um evento puramente empresarial com finalidade de trazer promoção e aumentar o fluxo de turistas para a região. 0 segundo evento é técnico científico e visa contribuir para o desenvolvimento de relacionamentos entre empresas, governo e universidades.

Quadro 2 - Clusters e suas características nos diferentes graus de maturidade

\begin{tabular}{|l|l|}
\hline \multicolumn{1}{|c|}{ Classificação } & \multicolumn{1}{|c|}{ Características } \\
\hline Cluster informal & $\begin{array}{l}\text { Predominância de empresas de pequeno porte, com baixa qualificação } \\
\text { dos atores críticos, adoção de tecnologias rudimentares, sem } \\
\text { representatividade no mercado externo. } \\
\text { Desempenho econômico sofrível e inconstante, com empresas que } \\
\text { competem marginalmente, pouca propensão a cooperação, não há o } \\
\text { entrelaçamento entre firmas }\end{array}$ \\
\hline Cluster em estágio intermediário: & $\begin{array}{l}\text { Predominância de empresas de pequeno e médio porte, podendo existir } \\
\text { e as tecnologias adotadas são relativamente atualizadas. Há posições } \\
\text { variáveis quanto ao desempenho e a propensão à cooperação é baixa, } \\
\text { assim como o entrelaçamento entre firmas e a efetiva cooperação. }\end{array}$ \\
\hline Cluster em estágio organizado: & $\begin{array}{l}\text { Acolhe uma grande diversidade de estruturas e características } \\
\text { empresariais, porém os atores críticos adotam práticas de gestão } \\
\text { modernas, com tecnologias atualizadas e, embora haja prevalência de } \\
\text { pequenas e médias empresas é freqüente a presença de gatekeepers e } \\
\text { de spillovers de conhecimento. Os níveis e potencial de cooperação são } \\
\text { médios, com iniciativas de desverticalização, porém ainda insuficientes } \\
\text { para gerar ganhos em flexibilidade produtiva e eficiência operacional }\end{array}$ \\
\hline d) clusters inovativos: & $\begin{array}{l}\text { Pressupõem a desverticalização da produção, a abertura de canais de } \\
\text { informação, a presença de gatekeepers e spillover de conhecimento e } \\
\text { um elevado grau de sinergia entre os diferentes atores do aglomerado }\end{array}$ \\
\hline
\end{tabular}

Fonte: Adaptado de Cunha (2003)

Com o levantamento de dados relacionados a aglomeração de organizações ligadas ao turismo e a cooperação interorganizacional no município de Balneário Camboriú foi utilizado o modelo proposto por Cunha (2003) (ver quadro 2) para nortear as análises. A seleção do modelo de análise foi motivada por sua inspiração nos critérios utilizados pela EURADA em suas pesquisas para classificação e avaliação do desempenho de aglomerados produtivos na União Européia, cujos clusters já se 
encontram consolidados e até em fase de reestruturação. Neste sentido, destaca-se a presença de dois importantes aspectos, a presença e atuação de gatekeepers de conhecimento (Giuliani, 2011; Guo \& Guo, 2011; Sohn et al., 2016). Em relação aos procedimentos de análise, a metodologia de Cunha (2003) classifica os clusters em quatro graus de maturidade: informais, intermediários, organizados ou inovativos.

\section{DESCRIÇÃO DOS ELEMENTOS CONS- TITUINTES E ANÁLISE DAS CARAC- TERÍSTICAS E ATRIBUTOS DO CLUSTER}

A cidade de Balneário Camboriú é conhecida como a Capital Catarinense do Turismo. O município conta com um alto nível de desenvolvimento, visto que de acordo com dados do Programa das Nações Unidas para o Desenvolvimento (PNUD) em parceria com o Instituto de Pesquisa Econômica Aplicada (IPEA), apresentou em 2013 o segundo melhor IDH no Estado, com um PIB per capita de $\mathrm{R} \$ 22.328$.

Localizada próximo a importantes cidades do estado de Santa Catarina, como Florianópolis, Blumenau e Joinville, conta com três aeroportos na proximidade (localizados em Florianópolis, Navegantes e Joinville). O acesso ao município dá-se pela BR-101 considerada uma das melhores rodovias do Brasil.

Conforme exposto na Figura 2, o município faz divisas com cidades que sediam importantes eventos em nível nacional, como a Oktoberfest (realizada em Blumenau) e a Volvo Ocean Race (realizada em Itajaí) e também de atrativos turísticos reconhecidos internacionalmente como o Beto Carrero World (localizado em Penha) e a casa noturna Green Valley (localizada em Camboriú).

Figura 2- Localização de Balneário Camboriú

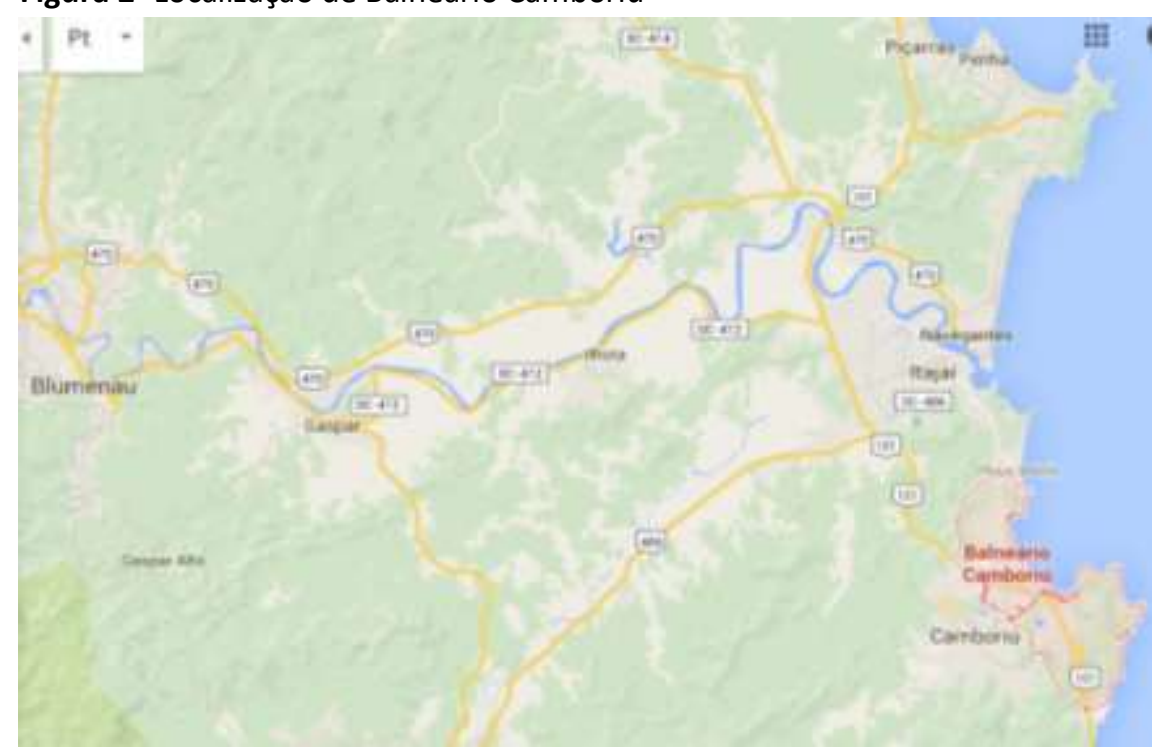

Fonte: Google Maps (2016)

Balneário Camboriú foi eleita a nona turismo no Brasil, de acordo com o Ministério cidade preferida por estrangeiros para o do Turismo (2015). Foi considerada um dos 
65 destinos indutores de turismo do Brasil (Brasil, 2014) e atende uma demanda turística de 600 mil visitantes por mês durante os meses de dezembro à fevereiro e aproximadamente $\mathbf{2 0 0}$ mil nos demais meses do ano (Prefeitura Municipal de Balneário Camboriú, 2016).

Para atender ao considerável número de visitantes durante o período de alta temporada, e minimizar os efeitos provocados pela sazonalidade turística, Balneário Camboriú desenvolveu uma relevante infraestrutura de produtos e serviços destinados a atender a necessidade da atividade turística, sendo esta composta por diversos hotéis, agências de turismo, prestadores de serviço de alimentação, de transporte, de eventos, entre outros, atendendo a demanda dos serviços voltados à saúde e bem estar, eventos, passeios naturais, passeios radicais, eventos culturais, e vida noturna estruturada (Prefeitura Municipal de Balneário Camboriú, 2015).

No que tange aos serviços de hospedagem disponibilizados em Balneário Camboriú, o município conta com mais de uma centena de empresas destinadas ao setor, estando divididas entre resorts, hostels, casas de excursão, pousadas e predominantemente hotéis. Ao total, o município conta com 7734 Unidades Habitacionais resultando em 19.034 leitos para atender aos seus visitantes (Prefeitura Municipal de Balneário Camboriú, 2015).

Quadro 3 - Meios de Hospedagem de Balneário Camboriú (SC)

\begin{tabular}{|c|c|}
\hline \multicolumn{2}{|c|}{ Meios de Hospedagem de Balneário Camboriú } \\
\hline Hotéis & 76 \\
\hline Resort & 25 \\
\hline Pousadas & 4 \\
\hline Hostels & 21 \\
\hline
\end{tabular}

Fonte: Plano Municipal de Turismo de Balneário Camboriú 2015-2025 (2015)

Uma vasta diversidade de Municipal de Turismo de Balneário Camboriú equipamentos de alimentos e bebidas é (2015) são 331 estabelecimentos (Quadro 4). encontrada na cidade. Conforme o Plano

Quadro 4 - Opções de alimentação disponíveis em Balneário Camboriú

\begin{tabular}{|c|c|}
\hline Serviço de alimentos e bebidas & 178 \\
\hline Restaurantes e bares & 90 \\
\hline Cafeterias/Confeitarias & 22 \\
\hline Churrascarias/ Galeterias/Grill & 41 \\
\hline Pizzarias & \\
\hline
\end{tabular}

Fonte: Plano Municipal de Turismo de Balneário Camboriú 2015-2025 (2015)

Em Balneário Camboriú estão atrativos culturais, eventos gastronômicos, localizadas 18 praças para atividades ao ar culturais e eventuais homenagens e 14 livre, 14 equipamentos de lazer, 10 recursos monumentos em homenagem a diversas 
lendas e personalidades de Balneário Camboriú.

O complexo de lazer noturno agrupa 106 estabelecimentos, sendo 82 bares e cervejarias e 10 casas noturnas (contando com algumas redes nacionais de festas).
Destaca-se a presença de casas noturnas como a Green Valley, eleita o segundo melhor clube de música eletrônica do mundo em 2016 pela revista DjMag, especializada na área (DjMag, 2016).

Quadro 5 - Serviços relacionados a Eventos em Balneário Camboriú

\begin{tabular}{|c|c|}
\hline \multicolumn{1}{|c|}{ Eventos em Balneário Camboriú } & 4 \\
\hline Organizadoras de Eventos & 5 \\
\hline Espaço para a realização de eventos & 82 \\
\hline Bares e Cervejarias & 7 \\
\hline Bares de esportes & 7 \\
\hline Boates prive & 10 \\
\hline Casas noturnas/danceterias & \\
\hline
\end{tabular}

Fonte: Plano Municipal de Turismo de Balneário Camboriú 2015-2025 (2015)

O turismo em Balneário Camboriú conta com passeios em meio a Mata Atlântica no percurso da Rodovia Interpraias, que liga Balneário Camboriú ao município vizinho, Itapema. Esta rodovia revela paisagens únicas com acesso a seis praias pertencentes ao município: Praia de Laranjeiras, Praia de Taquarinhas, Praia de Taquaras, Praia do Pinho, Praia do Estaleiro, Praia do Estaleirinho. A cidade oferece além das praias uma gama de atrativos turísticos como: Parque Unipraias, Parque Natural Raimundo Malta, Cristo Luz, Passarelas do Pontal Norte, Morro do Careca, Molhe da Barra Sul, Parque Cyro Gevaerd.

Balneário Camboriú possui 56 agências de viagens e turismo cadastradas no Cadastro Brasileiro de Empresas de Turismo [CADASTUR] em 2016, atuando como operadoras de turismo, agências emissivas de viagens e turismo e ainda, agências destinadas ao turismo receptivo na localidade. 0 município conta também com 5 organizadoras de eventos, havendo disponibilidade de 4 espaços para a realização destes (Prefeitura Municipal de Balneário Camboriú, 2015).

O município conta com 21 associações e 7 sindicatos composto pelas entidades relacionadas ao setor turístico, além de 74 guias de turismo. O município também conta com um considerável número de estabelecimentos de ensino superior (graduação, pós-graduação, MBA, mestrado e doutorado) somando dez instituições detentoras de um amplo número de cursos. Por meio da visita aos sites das instituições de ensino, verificou-se que todas disponibilizam cursos em áreas correlatas ao turismo, como administração, marketing, ciências contábeis e outras. É importante ressaltar que a Universidade do Vale do Itajaí [UNIVALI] disponibiliza formação em turismo e hotelaria para diferentes níveis, sendo estes: graduação, mestrado e doutorado. 
Quadro 6- Instituições de ensino em Balneário Camboriú

\begin{tabular}{|l|c|}
\hline \multicolumn{1}{|c|}{ Instituição } & $\begin{array}{c}\text { Dependência } \\
\text { Administrativa }\end{array}$ \\
\hline Faculdade AVANTIS e Instituto Superior de Educação AVANTIS & Privada \\
\hline Faculdade SOCIESC de Balneário Camboriú & Privada \\
\hline Universidade do Vale do Itajaí - UNIVALI - Balneário Camboriú & Privada \\
\hline Centro Universitário Leonardo Da Vinci - UNIASSELVI - Balneário Camboriú & Privada \\
\hline Faculdade do Litoral Catarinense & Privada \\
\hline Universidade Castelo Branco - Polo Balneário Camboriú & Privada \\
\hline Universidade Paulista - Polo Balneário Camboriú & Privada \\
\hline Universidade Anhembi Morumbi - Polo Balneário Camboriú & Privada \\
\hline Centro Universitário de Maringá - Polo Balneário Camboriú & Estadual \\
\hline Universidade do Estado de Santa Catarina7 - UDESC & \\
\hline
\end{tabular}

Fonte: Secretaria do Estado da Educação de Santa Catarina (2016)

Além da relevância relacionada aos aspectos de identidade e valorização cultural local e de opções de produtos e serviços, garantindo a concorrência e livre comércio local, o turismo é importante fomentador da economia de Balneário Camboriú, principalmente no que se refere à geração e oferta de emprego e renda para a comunidade local. Conforme exposto no quadro 7 , a atividade gera em média 10.254 mil empregos diretos (Prefeitura Municipal de Balneário Camboriú, 2015).

Cita-se que a Prefeitura de Balneário Camboriú (SC) é destaque na implementação de uma política formal de conscientização do turista sobre como respeitar a comunidade local/destino. São realizados programas de incentivo ao uso dos equipamentos turísticos pela população local, por órgãos municipais. Resultado desta política é evidenciado pelo relatório do índice de Competitividade do Turismo Nacional (Brasil, 2014) que dá destaque para a dimensão da cooperação regional, na qual, Balneário Camboriú obteve um dos melhores desempenhos dentre as cidades não capitais. A cooperação foi medida por meio da avaliação da governança regional, de projetos de cooperação regional, do planejamento turístico regional, da roteirização e promoção e apoio à comercialização de forma integrada. A média nacional de Cooperação regional alcançou 48,3 pontos e Balneário Camboriú obteve 70,1 pontos. No ranking geral Balneário Camboriú está entre as 15 melhores cidades turísticas do país, com 69,9 pontos. A pontuação está acima da média Brasil do estudo (59,5 pontos) e até mesmo da média das capitais $(68,2)$.

\footnotetext{
7 A UDESC em Balneário Camboriú não consta no website da Secretaria de Estado de Educação de Santa Catarina.
} 
Quadro 7- Empregos diretos gerados pelo turismo em Balneário Camboriú e média salarial

\begin{tabular}{|c|c|c|}
\hline \multicolumn{3}{|c|}{ Empregos ligados ao turismo em Balneário Camboriú } \\
\hline Setor & $\begin{array}{l}\text { Número de empregos } \\
\text { gerados }\end{array}$ & $\begin{array}{c}\text { Média } \\
\text { salarial/pessoa }\end{array}$ \\
\hline Transporte rodoviário de passageiros & 580 & $\mathrm{R} \$ 1575,30$ \\
\hline Outros transportes terrestres & 41 & $\mathrm{R} \$ 4712,50$ \\
\hline Atividades de transporte não específicas & 155 & $\mathrm{R} \$ 3156,67$ \\
\hline Transporte aquaviário & 73 & $\mathrm{R} \$ 2936,57$ \\
\hline Transporte aéreo & 21 & $\mathrm{R} \$ 1150,00$ \\
\hline $\begin{array}{l}\text { Atividades auxiliares dos transportes e atividades } \\
\text { relacionadas à organização do transporte de carga }\end{array}$ & 445 & $\mathrm{R} \$ 4971,16$ \\
\hline Alojamento & 1150 & $\mathrm{R} \$ 1409,78$ \\
\hline $\begin{array}{l}\text { Restaurantes e outros estabelecimentos de serviços de } \\
\text { alimentação e bebidas }\end{array}$ & 2745 & $\mathrm{R} \$ 1414,18$ \\
\hline $\begin{array}{l}\text { Serviços de catering, Buffet e outros serviços de comida } \\
\text { preparada }\end{array}$ & 300 & $\mathrm{R} \$ 1316,90$ \\
\hline Serviços ambulantes de alimentação & 31 & $\mathrm{R} \$ 1766,67$ \\
\hline Atividades de alimentação não especificadas & 704 & $\mathrm{R} \$ 1513,75$ \\
\hline $\begin{array}{l}\text { Atividades cinematográficas, produção de vídeos e de } \\
\text { programas de televisão, gravação de som e de música }\end{array}$ & 62 & $\mathrm{R} \$ 1433,33$ \\
\hline Atividades imobiliárias & 1088 & $\mathrm{R} \$ 2519,62$ \\
\hline $\begin{array}{l}\text { Agências de viagens, operadores turísticos e serviços de } \\
\text { reservas }\end{array}$ & 311 & $\mathrm{R} \$ 1634,33$ \\
\hline Atividades de tele atendimento & 31 & $\mathrm{R} \$ 3000,00$ \\
\hline $\begin{array}{l}\text { Atividades de organização de eventos, exceto culturais e } \\
\text { esportivos }\end{array}$ & 21 & $\mathrm{R} \$ 1518,50$ \\
\hline $\begin{array}{l}\text { Outras atividades de serviços prestados principalmente às } \\
\text { empresas }\end{array}$ & 228 & $\mathrm{R} \$ 1932,86$ \\
\hline Outras atividades de ensino & 352 & $\mathrm{R} \$ 1603,24$ \\
\hline Atividades de educação não especificadas & 352 & $\mathrm{R} \$ 1929,41$ \\
\hline Atividades artísticas, criativas e de espetáculos & 580 & $\mathrm{R} \$ 2215,93$ \\
\hline Atividades ligadas ao patrimônio cultural e ambiental & 10 & $R \$ 2000,00$ \\
\hline Atividades de exploração de jogos de azar e apostas & 62 & $\mathrm{R} \$ 1750,00$ \\
\hline Atividades esportivas & 218 & $\mathrm{R} \$ 2334,29$ \\
\hline Atividades de condicionamento físico & 228 & $\mathrm{R} \$ 1997,73$ \\
\hline Atividades de recreação e lazer & 466 & $\mathrm{R} \$ 1584,00$ \\
\hline
\end{tabular}

Fonte: Plano Municipal de Turismo de Balneário Camboriú 2015-2025 (2015)

Destaca-se a implementação de ações estratégicas que visam estimular a cooperação regional e os ganhos coletivos. Neste sentido aponta-se dois importantes eventos para cidade: Balneário Saboroso e o Fórum Científico de Gastronomia, Turismo e Hotelaria (FCGTURH).

O Balneário Saboroso em 2016 teve sua sétima edição e é um evento promovido pelo Balneário Camboriú Convention \& Visitors Bureau. O evento tem como objetivo promover o turismo regional por meio da gastronomia local, trazendo maior visibilidade para o destino. Sua última edição contou com a participação de 38 bares e restaurantes oferecendo um menu completo com entrada, prato principal e sobremesa, por um preço único tabelado. Entre os 
apoiadores do evento estão importantes empresas localizadas na região e que atuam em diferentes setores, a Prefeitura da cidade, entidades como o SEBRAE Santa Catarina, e instituições de ensino como a UNIVALI. Os resultados da prestação de contas da sétima edição, que aconteceu em julho de 2016, apresentou um número recorde de pratos vendidos, foram 14.183 menus comercializados.

Em sua sétima edição o evento contou com duas novidades, uma ação social de arrecadação de alimentos e a presença do serviço de cliente oculto. Neste sentido por meio da parceria com o Lions Clube de Balneário Camboriú foram arrecadados mais de $200 \mathrm{~kg}$ de alimentos não perecíveis, todos doados ao Centro Especializado em Reabilitação de Toxicômanos e Alcoolistas de Camboriú. A participação da empresa Mister $O$, que trabalha com o serviço de cliente oculto levantou informações sobre a qualidade do menu, e revelou que o ponto mais bem avaliado foi a relação custobenefício. Isto devido a boa qualidade dos pratos, menus criativos e programação paralela atrativa (Balneário Saboroso, 2016). Diante do exposto, pode-se considerar que o evento Balneário Saboroso é uma ação que gera economias de aglomeração para empresas envolvidas.

Diante das evidências apresentadas consta-se que o Balneário Camboriú Convention \& Visitors Bureau por meio do Balneário Saboroso estimula a cooperação e a articulação positiva entre firmas que atuam em um mesmo segmento, promovendo a coopetição (Nalebuff \& Brandenburger, 2011). Os resultados observados ao longo das quatro edições evidenciam que o evento produz economias externas, geradas pela presença de três fatores: disponibilidade de inputs locais, mão de obra qualificada, e spillovers de conhecimento (Giuliani, 2011).

A integração entre os atores também por ser observada com eventos de cunho acadêmico que acontecem na mais importante universidade do cluster, a Universidade do Vale do Itajaí (UNIVALI). Neste sentido, cita-se o Fórum Científico de Gastronomia, Turismo e Hotelaria (FCGTURH) que teve início no ano de 2012. O objetivo do FCGTURH é propiciar a discussão do desenvolvimento turístico aliado a hotelaria e a gastronomia em todas as esferas, possibilitando o desenvolvimento e a integração dos diversos envolvidos com a área, dinamizando a integração entre a academia, a sociedade e o governo e socializando teorias do turismo e da gastronomia. Participam do evento pesquisadores das áreas do turismo, hotelaria e gastronomia do Brasil e do exterior que socializam os resultados de suas pesquisas e discutem tendências nestas áreas. Desde sua primeira edição o FCGTURH abre espaço para comunidade de CT\&l interessada em discutir temáticas relacionadas a governança e desenvolvimento regional e para empresas que queiram conhecer pesquisas das áreas de turismo, hotelaria e gastronomia a fim de aplicá-las em seu nicho de mercado. Durante as últimas edições do evento além da organização vinculada a Universidade do Vale do Itajaí (UNIVALI) também apoiaram o Fórum importantes instituições do trade turístico localizadas na cidade de Balneário 
Camboriú e importantes órgãos governamentais que apoiam a pesquisa e o desenvolvimento tecnológico como a Coordenação de Aperfeiçoamento de Pessoal de Nivel Superior [CAPES], o Conselho Nacional de Desenvolvimento Científico e Tecnológico [CPNQ], o Ministério da Educação e a Associação Brasileira de Turismólogos e Profissionais do Turismo [ABBTUR].

Em edições anteriores o Fórum contou com a participação de renomados pesquisadores na área de Turismo e Hotelaria como o Prof. Phd. Jafar Jafari (Winsconsin University EUA), Prof. Dr. João Albino Matos da Silva (Universidade do Algarve Portugal), Prof. Phd. Miguel Moital (Bournermouth University Inglaterra) e Profa Phd. Anita Eves (Surrey University - UK) que apresentaram conferências sobre Inovação em Gastronomia, permitindo um ambiente propício para a disseminação de conhecimento e oportunidade de discussão acerca da realidade do desenvolvimento do turismo local, regional e estadual, apoiado na gastronomia.

Constata-se que diante de sua atuação na formação de mão de obra especializada para o setor do turismo e por meio do FCGTURH a UNIVALI é considerada uma gatekeeper de conhecimento no cluster turístico de Balneário Camboriú. Observa-se que a instituição demonstra a capacidade de reconhecer os novos conhecimentos que provém do ambiente externo e contribui para disseminá-lo a fim de que seja aplicado para fins comerciais (Giuliani, 2011; Hortelano, Pérez, \& Villaverde, 2015). O Fórum também promove o spillover de conhecimento por meio de interações (Kesidou \& Romijn, 2008). Através de uma análise global dos eventos estudados, os pesquisadores consideram que o Balneário Saboroso e o FCGTURH criam e fortalecem plataformas para cooperação entre corporações e instituições para geração de conhecimento para o setor turístico (Borkowska-Niszczota, 2015).

Como constatações gerais destaca-se que:

- em relação ao evento Balneário Saboroso, a criação de um novo menu e o incremento nas vendas, resultados do evento Balneário Saboroso evidenciam impactos positivos da coopetição que ocorre no interior do cluster. Dentre estes impactos cita-se: o estímulo à inovação e o aumento da produtividade das empresas e o conhecimento das tendências de mercado (Iordache et al., 2010; Guo \& Guo, 2011; Sohn et al., 2016).

- em relação ao FCGTUHR, a UNIVALI desenvolve contatos com fontes externas de conhecimentos, como por exemplo, os pesquisadores de instituições localizadas no exterior (ex.: Portugal, Espanha, Inglaterra) atuando como gatekeeper de conhecimento no cluster turístico de Balneário Camboriú promovendo a formação de linkages intra e extra cluster (Giuliani, 2011) .

- ambos os eventos promovem a aprendizagem coletiva e podem ser classificados como mecanismos de transferência de conhecimento do cluster (Giuliani, 2011; Graf, 2011).

Os resultados apresentados retratam a presença de um aglomerado de empresas, organizações e uma infraestrutura que atuam 
no setor do turismo localizadas em Balneário Camboriú, e que existe indícios de cooperação interorganizacional visando ganhos coletivos e o desenvolvimento do setor na região. Baseado nos conceitos de Tomazzoni (2015), Martins et al., (2016), e Polukhina, (2016) os autores confirmam a existência de um cluster turístico na cidade de Balneário Camboriú.

Diante do exposto e com base no modelo de análise de clusters proposto por Cunha (2003) considera-se Balneário Camboriú um cluster em estágio intermediário com a atividade turística trazendo impactos positivos para a região. Percebe-se que há uma propensão a cooperação. Neste sentido pode-se também identificar no cluster características associadas a aglomerados organizados com a presença de gatekeepers e spillovers de conhecimento (Giuliani, 2011).

\section{CONSIDERAÇÕES FINAIS}

Como resultado considera-se que Balneário Camboriú é um cluster turístico, os dados levantados confirmam a presença de elementos constitutivos de clusters turísticos destacando-se a proximidade geográfica e cooperação dos seguintes atores críticos: empresas (meios de hospedagem, serviços de alimentos e bebidas), entidades governamentais, universidades e instituições de apoio, equipamentos de lazer, agências e atrativos turísticos.

O cluster turístico de Balneário Camboriú propicia condições favoráveis para a geração de vantagens competitivas coletivas e o fortalecimento do turismo na região. Desta forma, destaca-se a implementação de ações estratégicas que visam estimular a colaboração como os eventos "Balneário Saboroso" e o "Fórum Científico de Gastronomia, Turismo e Hotelaria (FCGTURH)".

A partir desta pesquisa surgiram diversas descobertas que conduziram os pesquisadores em busca das respostas sintetizadas nas conclusões. O trabalho de pesquisa ao mesmo tempo que elucida questionamentos, amplia a visão dos pesquisadores trazendo à tona novas possibilidades e com elas oportunidades de pesquisa. Estas oportunidades podem estar relacionadas ao aprimoramento da técnica de pesquisa adotada, do modelo de análise. Neste sentido, visando o aprimoramento da metodologia de análise adotada neste artigo sugere-se a sua aplicação em outros casos de clusters turísticos no Brasil e no exterior. Sugere-se também outros estudos sobre o cluster turístico de Balneário Camboriú, como por exemplo, uma análise de sua dinamicidade.

Finalmente, deve-se destacar que os resultados obtidos em pesquisas como esta, não podem ser generalizáveis pela própria natureza do tema e da metodologia utilizada, mas servem de insights a considerações que podem ser estendidas a outros clusters e modalidades de redes interorganizacionais no setor do turismo.

\section{REFERÊNCIAS}

Aleksandrova, A. Y. (2016). Typology of countries of the world according to the development level of international tourism. Geography and Natural Resources, 37(1), 18-25. 
Ashton, M. S. G., Valduga, V., \& Tomazzoni, E. L. (2015). Turismo criativo e desenvolvimento da oferta turística do cluster do Vale dos Vinhedos (RS, Brasil). Investigaciones Turísticas, (10), 90116.

Balneário Saboroso (2016) Convention Bureau realiza prestação de contas do 70 Balneário Saboroso. Recuperado em 10 set, 2016, de http://www.balneariosaboroso.com.br/saboroso 2016/noticias/titulo/convention-bureau-realizaprestacao-de-contas-do-7-balneario-saboroso/.

Beni, M. C. (2003). Globalização do turismo: megatendências do setor e a realidade brasileira (1a ed.). São Paulo: Aleph.

Borkowska-Niszczota, M. (2015). Tourism clusters in Eastern Poland-analysis of selected aspects of the operation. Procedia-Social and Behavioral Sciences, 213, 957-964.

Brandenburger, A. M. \& Nalebuff, B. J. (2011). Coopetition. New York: Crown Business.

Brasil, Ministério do Turismo (2015) Estudo da Demanda Turística Internacional. Brasília: MTUR. Recuperado em 20 maio, 2016, de http://www.dadosefatos.turismo.gov.br/dadose fatos/demanda_turistica/internacional/

Brasil, Ministério do Turismo (2014) Índice de competitividade do Turismo Nacional: Relatório Brasil 2014. Brasília: MTUR. Recuperado em 20 maio, 2016, de http://www.dadosefatos.turismo.gov.br/export/ sites/default/dadosefatos/outros_estudos/down loads_outrosestudos/Relatorio_Brasil_2014_we b.pdf.

Cunha, I. J. (2003) Aglomerados industriais de economias em desenvolvimento: classificação e caracterização. Florianópolis: Edeme.

Cunha, S. K. D., \& Cunha, J. C. D. (2005). Tourism cluster competitiveness and sustainability: proposal for a systemic model to measure the impact of tourism on local development. $B A R$ Brazilian Administration Review, 2(2), 47-62.
Cunha, I. J. (2007) Governança, Internacionalização e competitividade de aglomerados produtivos de móveis no Sul do Brasil, Portugal e Espanha. Chapecó: Arcus Ind. Gráfica.

DjMag (2016) Top 100 world clubs. Recuperado em 20 maio, 2016, de https://www.djmag.com/top100clubs.

Elche-Hortelano, D., Martínez-Pérez, Á., \& GarcíaVillaverde, P. M. (2015). Bonding capital, explotación de conocimiento e innovación incremental en los clusters de turismo cultural: las Ciudades Patrimonio de la Humanidad en Espana. Investigaciones Europeas de Dirección y Economía de la Empresa,21(3), 120-128.

Ferreira, J., \& Estevao, C. (2009). Regional competitiveness of a tourism cluster: A conceptual model proposal. Encontros científicos, Tourism \& management studies, 37-51.

Fundeanu, D. D. (2015). Innovative Regional Cluster, Model of Tourism Development. Procedia Economics and Finance, 23, 744-749.

Gerhardt, T. E., \& Silveira, D. T. (2009) Métodos de Pesquisa. Porto Alegre: Editora da UFRGS. Recuperado em 20 maio, 2016, de http://www.ufrgs.br/cursopgdr/downloadsSerie /derad005.pdf.

Giuliani, E. (2011) Role of technological gatekeepers in the growth of industrial clusters: Evidence from Chile. Regional Studies, 45(10), 1329-1348.

Guo, B., \& Guo, J. (2010) Patterns of technological learning within the knowledge systems of industrial clusters in emerging economies: Evidence from China. Technovation, 37(2), 87104.

Guo J., \& Guo B. (2011) How do innovation intermediaries facilitate knowledge spillovers within industrial clusters? Asian Journal of Technology Innovation, 21(2), 31-49. 
Graf, H. (2011). Gatekeepers in regional networks of innovators. Cambridge Journal of Economics, 35(1), 173-198.

Kesidou, E., \& Romijn, H. (2008) Do Local Spillovers Matter For Development? An Empirical Study of Uruguay's Software Cluster. World Development, 36(10), 2004-2028.

Kunz, J. G., Schommer, L., Schneider, M., \& Mecca, M. S. (2012). A Clusterização do Turismo em Gramado-RS: Breves Notas. Revista Rosa dos Ventos, 4, 609-614.

lordache, C., Ciochinã, I. \& Asandei, M. (2010) Clusters - Tourism Activity Increase Competitiveness Support. Theoretical and Applied Economics. 18(5), 99-112.

Jackson, J., \& Murphy, P. (2006). Clusters in regional tourism An Australian case. Annals of Tourism research, 33(4), 1018-1035.

Lai, Y. L., Hsu, M. S., Lin, F. J., Chen, Y. M., \& Lin, Y. H. (2014). The effects of industry cluster knowledge management on innovation performance. Journal of Business Research, 67(5), 734-739.

Lubeck, R. M., Wittmann, M. L., \& Silva, M. S. (2012). Afinal, quais variáveis caracterizam a existência de cluster Arranjos Produtivos Locais (APLS) e dos Sistemas Locais De Produção E Inovação (SLPIS)?. Revista Ibero-Americana de Estratégia, 11(1), 120-151.

Mabrouk, F., McDonald, M., Mocan, S., \& Summa, T. (2008). The Tunisian Tourism Cluster. Harvard Business School, Boston, MA.

Martins, C., Fiates, G. G. S., \& Pinto, A. L. (2016). A relação entre os clusters de turismo e tecnologia e seus impactos para o desenvolvimento local: um estudo bibliométrico da produção científica. Revista Brasileira de Pesquisa em Turismo, 10(1), 65-88.

Menzel, M. P., \& Fornahl, D. (2009). Cluster life cycles-dimensions and rationales of cluster evolution. Industrial and corporate change, dtp036.

Morosini, P. (2004). Industrial clusters, knowledge integration and performance. World development, 32(2), 305-326.

Novelli, M., Schmitz, B., \& Spencer, T. (2006). Networks, clusters and innovation in tourism: $A$ UK experience. Tourism management,27(6), 1141-1152.

Polukhina, A. (2016) A cluster model of ethnotourism organization in Russian regions. Worldwide Hospitality and Tourism Themes, v. 8 n. 3, pp. $359-372$.

Porter, M. (2008, January). Clusters, innovation, and competitiveness: New findings and implications for policy. In Presentation given at the European Presidency Conference on Innovation and Clusters in Stockholm (Vol. 23).

Porter, M.E., \& Kramer, M.R. (2011) Criação de valor compartilhado: como reinventar o capitalismo e desencadear uma onda de inovação e crescimento, In: Harvard BusinessReview, 89(1), 21-33.

Prefeitura Municipal de Balneário Camboriú (2015) Plano Municipal de Turismo. Balneário Camboriú, SC, Brasil. Recuperado em 20 maio, 2016, de http://www.balneariocamboriu.sc.gov.br/impre nsa/noticia.cfm ?codigo $=13575$.

Prodanov, C. C., \& Freitas, E. C. de (2013) Metodologia do Trabalho Científico: Métodos e Técnicas da Pesquisa e do Trabalho Acadêmico (2a ed.) Novo Hamburgo: Editora Feevale. Recuperado em 20 maio, 2016, de http://www.faatensino.com.br/wpcontent/uploads/2014/11/2.1-E-bookMetodologia-do-Trabalho-Cientifico-2.pdf.

Programa das Nações Unidas para o desenvolvimento [PNUD] (2013) Atlas do desenvolvimento humano do Brasil. Recuperado em 20 maio, 2016, de 
http://www.pnud.org.br/atlas/.

Santos, S. V., \& Cerdeira, J. P. (2013). Estratégias de coopetição em Portugal: $O$ contributo dos polos e clusters de empresas. Exedra: Revista Científica, (8), 22-34.

Silva, P. M. da (2014) Aglomerados e turismo: análise da produção científica nacional e internacional sobre o tema. Revista Turismo Visão e Ação, 16(2).

Secretaria de Educação do Estado de Santa Catarina (2016) Portal da educação Institucional. Recuperado em 02 agosto, 2016, de http://serieweb.sed.sc.gov.br/cadiesportal.aspx.

Sohn, A. P. L., Vieira, F. D., Casarotto Filho, N. C., Cunha, I. J., \& Zarelli, P. R. (2016). Knowledge Transmission in Industrial Clusters: Evidence from EuroClusTex. European Planning Studies, 24(3), 511-529.

Souza, D. A., \& Gil, A. C. (2015). A Importância da Identidade Regional na Configuração de Clusters Turísticos. Revista Turismo em Análise, 26(2). Souza, D. A., \& Gil, A. C. (2014) Produção científica nacional sobre clusters turísticos . Revista Turismo - Visão e Ação, 16(3).

The Travel \& Tourism Competitiveness Report 2015. Recuperado em 10 set, 2016, de http://www3.weforum.org/docs/TT15/WEF_Glo bal_Travel\&Tourism_Report_2015.pdf.

Tomazzoni, E. L., \& da Costa, J. S. (2015). Ações estratégicas e visões dos atores do cluster de turismo da cidade de São Paulo. Revista Brasileira de Pesquisa em Turismo, 9(1), 3-21.

Yıldız, T., \& Aykanat, Z. (2015). Clustering and Innovation Concepts and Innovative Clusters: An Application on Technoparks in Turkey. ProcediaSocial and Behavioral Sciences, 195, 1196-1205.
Waxell, A., \& Malmberg, A. (2007). What is global and what is local in knowledge-generating interaction? The case of the biotech cluster in Uppsala, Sweden. Entrepreneurship and Regional Development, 19(2), 137-159.

Wittmann, M. L., \& da Silva, M. S. (2012). AFinal, quais variáveis caracterizam a existência de cluster arranjos produtivos locais (apls) e dos sistemas locais de produção e inovação (SLPIS)?. Revista Ibero-Americana de EstratégiaRIAE, 11(1), 120-151.

World Tourism Organization (n.d) Why tourism? Recuperado em 08 novembro, 2016, de http://www2.unwto.org/content/why-tourism.

\section{Dados dos Autores}

\section{Ana Paula Sohn}

Doutora em Engenharia da Produção pela Universidade Federal de Santa Catarina (UFSC). Professora e pesquisadora no Programa de PósGraduação em Turismo e Hotelaria da Universidade do Vale do Itajaí (UNIVALI). E-mail: anasohn@hotmail.com

\section{Carolina Silvestrini}

Graduanda em Design na Universidade do Vale do Itajaí (UNIVALI). Bolsista do Programa Institucional de Bolsas de Iniciação Cientifica (PROBIC). E-mail: c.silvestrini@hotmail.com

\section{Thamires Foletto Fiuza}

Mestre pelo Programa de Pós-Graduação em Turismo e Hotelaria da Universidade do Vale do Itajaí (UNIVALI). Bolsista da Coordenação de Aperfeiçoamento de Pessoal de Nível Superior (CAPES). E-mail: thamires.fiuza@edu.univali.br

\section{Pablo Flôres Limberger}

Doutor em Turismo e Hotelaria pela Universidade do Vale do Itajaí (UNIVALI). Professor e pesquisador no Programa de Pós-Graduação em Turismo e Hotelaria da Universidade do Vale do Itajaí (UNIVALI). E-mail: pablofı@univali.br 